\title{
Practice patterns to decrease myopia progression differ among paediatric ophthalmologists around the world
}

Ari Leshno ${ }^{1,2}$, Sonal K Farzavandi ${ }^{3}$, Rosario Gomez-de-Liaño ${ }^{4}$, Derek T Sprunger ${ }^{5}$, Tamara Wygnanski-Jaffe ${ }^{1,2}$, Eedy Mezer ${ }^{6,7}$

1. Ophthalmology, Sheba Medical Center at Tel Hashomer, Tel Hashomer, Israel

2. Sackler Faculty of Medicine, Tel Aviv University, Tel Aviv, Israel

3. Ophthalmology, Singapore National Eye Centre, Singapore

4. Hospital Clínico/Instituto Castroviejo, Universidad Complutense de Madrid, Madrid, Comunidad de Madrid, Spain

5. Department of Ophthalmology, Pediatric and Strabismus Section, Indiana University School of Medicine, Indianapolis, Indiana, USA

6. Ophthalmology, Ruth Rappaport Children's Hospital, Rambam Health Care Campus, Haifa, Israel

7. Ophthalmology, Bruce and Ruth Rappaport Faculty of Medicine, Technion Israel Institute of Technology, Haifa, Israel

Correspondence to Dr Eedy Mezer, Ophthalmology, Ruth Rappaport Children's Hospital, Rambam Health Care Campus, Haifa, Israel; emezer@technion.ac.il

\section{ABSTRACT}

Introduction Myopia is a worldwide epidemic. Plethora of treatments are offered to decrease myopia progression. In this study, we compared between different geographical areas worldwide the practice patterns used by paediatric ophthalmologists to decrease the progression of myopia.

Methods Global responses to a questionnaire were analysed $(n=794)$ for demographic variations. Pharmacological, optical and behavioural categories were defined as effective or ineffective based on the current scientific peer reviewed literature.

Results Treatment rates varied significantly between geographical regions (mean $57 \%$, range $39 \%-89 \%, p<0.001)$. Nearly all participants who treat myopia used at least one form of effective treatment, regardless of location $(98 \%, p=0.16)$. Among those prescribing pharmacological treatments, European physicians offered the lowest rate of effective treatment compared with other regions ( $85 \%$ vs mean $97 \%$ ). Rates of effective optical treatment varied significantly

This is the author's manuscript of the article published in final edited form as:

Leshno, A., Farzavandi, S. K., Gomez-de-Liaño, R., Sprunger, D. T., Wygnanski-Jaffe, T., \& Mezer, E. (2020). Practice patterns to decrease myopia progression differ among paediatric ophthalmologists around the world. British Journal of Ophthalmology, 104(4), 535-540. https://doi.org/10.1136/bjophthalmol-2019-314752 
between locations ( $p<0.001$ ), from 16\% (Central-South America) to 56\% (Far East). Most treating respondents advocated behavioural modifications (92\%), between 87\% (North America) and 100\% (Central Asia). Nearly all respondents used combinations of treatment modalities (95\%)— mostly pharmacological, optical and behavioural combination. However, combination rates varied significantly between regions $(p<0.001)$.

Discussion The utility of treatment to decrease myopia progression differs significantly across the world both in type, combination and efficacy.

Conclusion Paediatric ophthalmologists involvement and proficiency in myopia progression treatment varies around the world. This may entail promoting continuous medical education and other incentives to increase the number and proficiency of paediatric ophthalmologist to have a more effective impact to control the myopia epidemic in children.

Keywords: child health (paediatrics); optics and refraction; public health; treatment medical

\section{INTRODUCTION}

Myopia is a well-known cause of ocular morbidity and is considered to be a major risk factor for vision impairment and sight-threatening complications. $\frac{12}{2}$ Myopia is the most common visual disorder with increasing prevalence rates worldwide. Lately, the WHO has declared myopia as an epidemic. $\frac{3}{}$ The magnitude of the phenomena and socioeconomic burden derived from this condition led to the development of various strategies to control myopia by either pharmacological, behavioural or optical measures. Although a consensus regarding the best treatment approach has not yet been determined, several treatments were proven to be effective. $\frac{45}{5}$ However, in absence of an official treatment protocol, there is a wide variation in treatment patterns among paediatric ophthalmologists who have chosen to treat children and teenagers to decrease myopia progression. $\underline{6}$

The purpose of this study was to map the practice patterns to control myopia progression in the different world regions. 


\section{METHODS}

\section{DATA COLLECTION}

Responses regarding the approach to control myopia progression were collected from paediatric ophthalmologists worldwide using an online survey between December 2016 and June 2017 as outlined previously. $\underline{6}$ The questionnaire included 17 questions related to characteristics of the paediatric ophthalmologists, the myopic patient population and choice of treatment modalities to halt the progression of myopia.

\section{EFFECTIVE TREATMENT DEFINITION}

Treatments were deemed effective or ineffective based on a previous analysis as long as they statistically significantly decreased the progression of myopia. $\frac{457-12}{4}$ Treatments derived from online responses were divided into three categories: pharmacological, optical or behavioural. For every respondent, each treatment group was classified as effective if the respondent employed at least one effective method within that group to reduce myopia progression.

Table 1

Characteristics of participants treating myopia

\begin{tabular}{|c|c|c|c|c|c|c|c|c|}
\hline gion & $\begin{array}{c}\text { North } \\
\text { America } \\
(\mathrm{n}=151)\end{array}$ & $\begin{array}{l}\text { Central- } \\
\text { South } \\
\text { America }\end{array}$ & $\begin{array}{l}\text { Europe } \\
(\mathrm{n}=51)\end{array}$ & $\begin{array}{c}\text { Middle } \\
\text { East } \\
(\mathrm{n}=34)\end{array}$ & $\begin{array}{l}\text { Central } \\
\text { Asia } \\
(n=65)\end{array}$ & $\begin{array}{c}\text { Far } \\
\text { East } \\
(n=91)\end{array}$ & $\begin{array}{c}\text { Australia } \\
(\mathrm{n}=16)\end{array}$ & $\begin{array}{l}\text { Total } \\
(\mathrm{n}=455)\end{array}$ \\
\hline
\end{tabular}

Affiliation

\begin{tabular}{|c|c|c|c|c|c|c|c|c|}
\hline & 49 & 17 & 24 & 20 & 22 & 49 & 9 & 190 \\
\hline $\begin{array}{c}\text { University } \\
\text { hospital }\end{array}$ & $32.5 \%$ & $36.2 \%$ & $47.1 \%$ & $58.8 \%$ & $33.8 \%$ & $53.8 \%$ & $56.3 \%$ & $41.8 \%$ \\
\hline
\end{tabular}




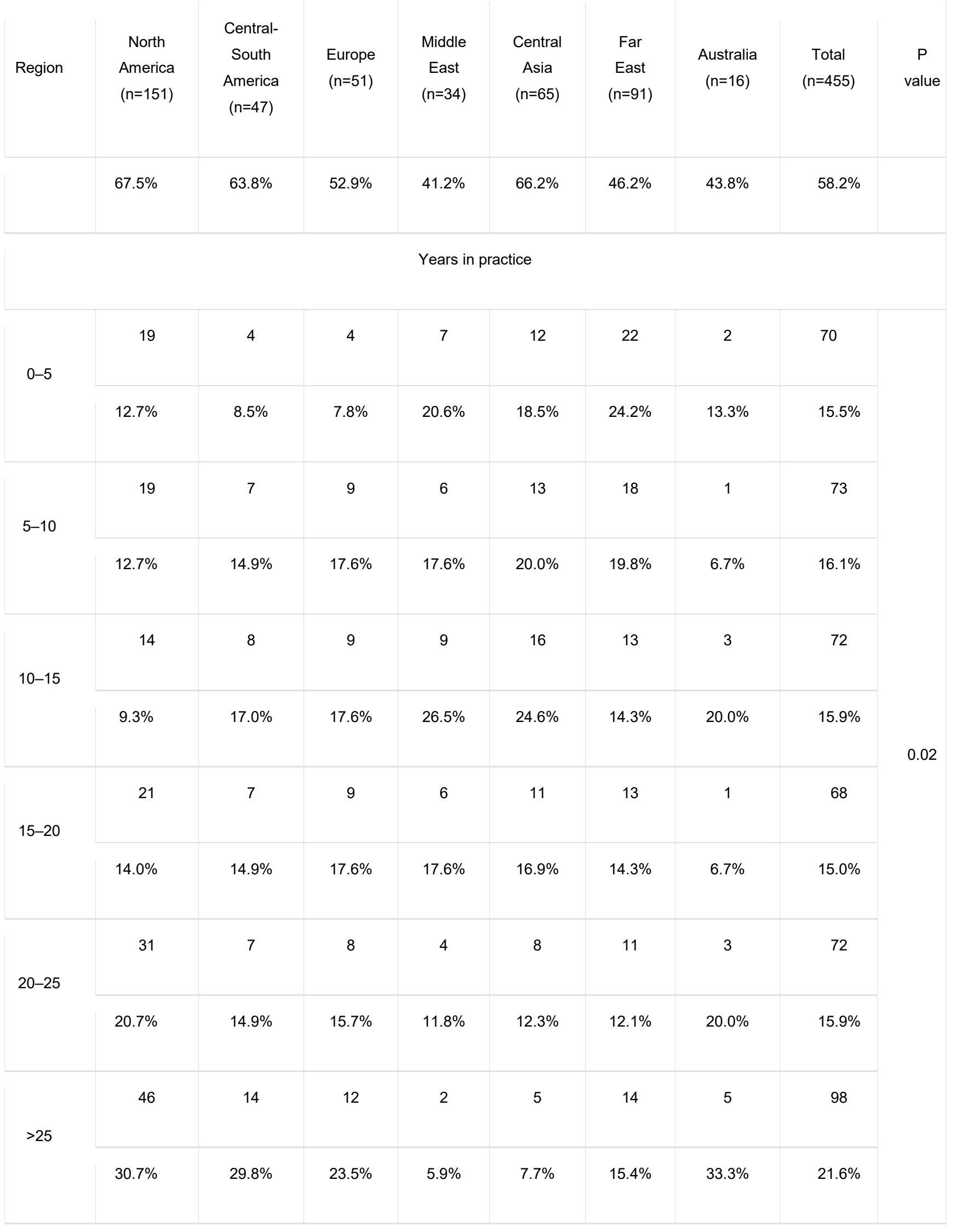




\begin{tabular}{|c|c|c|c|c|c|c|c|c|c|}
\hline Region & $\begin{array}{c}\text { North } \\
\text { America } \\
(n=151)\end{array}$ & $\begin{array}{c}\text { Central- } \\
\text { South } \\
\text { America } \\
(n=47)\end{array}$ & $\begin{array}{l}\text { Europe } \\
(n=51)\end{array}$ & $\begin{array}{c}\text { Middle } \\
\text { East } \\
(n=34)\end{array}$ & $\begin{array}{c}\text { Central } \\
\text { Asia } \\
(n=65)\end{array}$ & $\begin{array}{c}\text { Far } \\
\text { East } \\
(n=91)\end{array}$ & $\begin{array}{c}\text { Australia } \\
(n=16)\end{array}$ & $\begin{array}{c}\text { Total } \\
(n=455)\end{array}$ & $\begin{array}{c}\mathrm{P} \\
\text { value }\end{array}$ \\
\hline
\end{tabular}

Experience in myopia treatment (years)

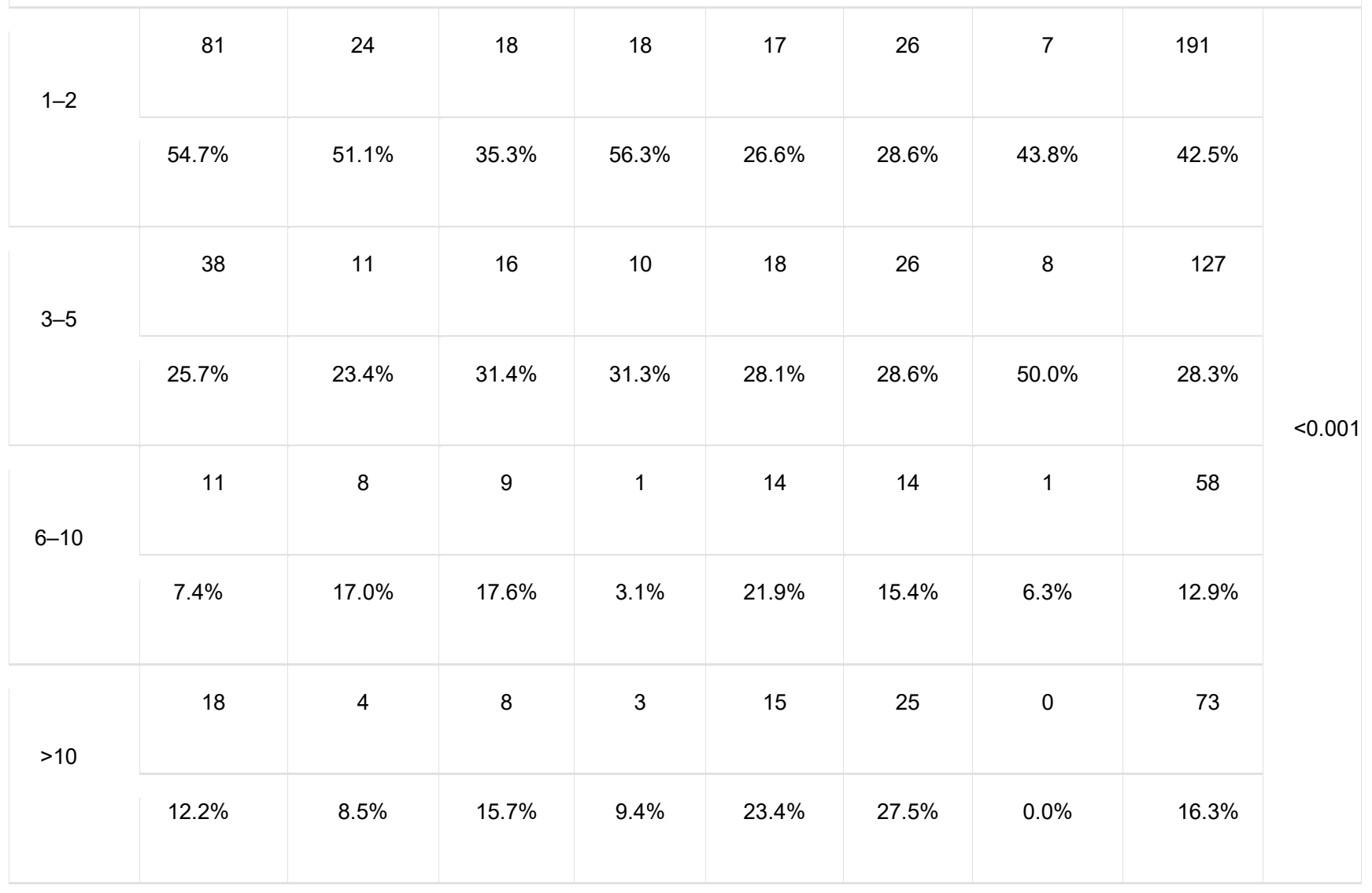

\section{STATISTICAL ANALYSIS}

Data were analysed by statistical analysis software (IBM SPSS for windows, V.25). Significance was defined as an alpha error probability $<0.05$. The $X^{2}$ test was used for comparison of categorical variables and Student's $t$-test and one-way analysis of variance (ANOVA) were used for comparison of continuous variables. Linear and binary logistic regressions analysis were applied as needed to detect interactions between variables and exclude confounder effects. 
All authors declared no financial or non-financial conflict of interest, and no formal consents were required as the information obtained through the questionnaire did not refer to a specific patient but rather to the general clinical treatment patterns of the treating ophthalmologist.

The study adhered to the principles outlined in the Declaration of Helsinki and was approved by the local institutional review board (IRB) at Sheba Medical Center, Tel Hashomer, Israel.

\section{RESULTS}

\section{RESPONDENT'S CHARACTERISTICS}

Of the 794 responses received from paediatric ophthalmologists worldwide, 455 (57.3\%) were from those who have chosen to treat to reduce the progression of myopia. The responses were grouped into seven geographical regions (figure 1). The relative size of groups from various geographical regions varied significantly between all the respondents and those that treat to reduce myopia progression. Physicians from North America and the Far East comprised a larger part of the treating group than in the group of all the respondents. In contrast, the relative part of physicians from Europe in the treating group was smaller than in all the respondents. Distribution of university affiliation, overall duration of experience to treat to reduce myopia progression and length of practice in years were similar to that of the general cohort. 


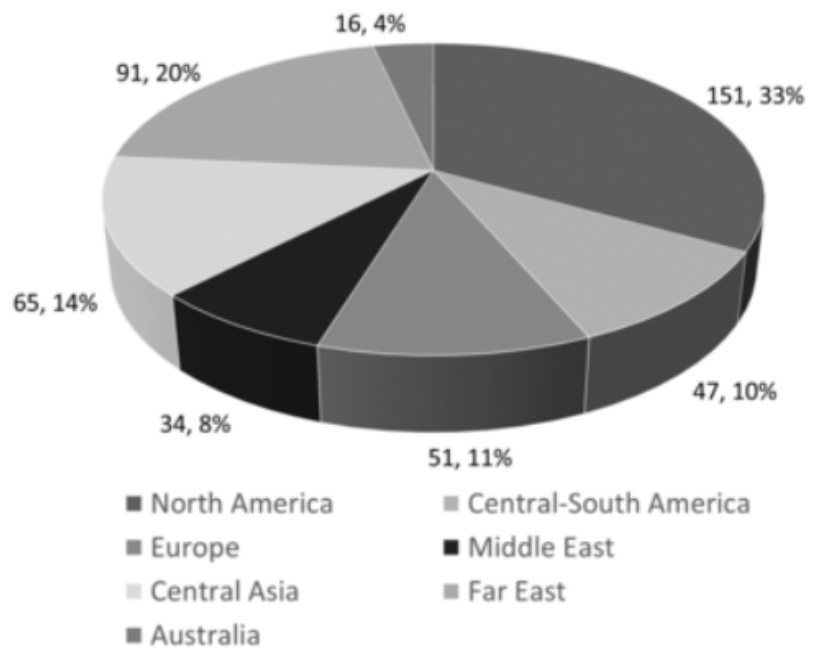

\section{FIGURE 1}

\section{DISTRIBUTION OF RESPONDENTS BY GEOGRAPHICAL REGION.}

Respondents' characteristics are summarized in table 1. North American ophthalmologists comprised the largest group (151, 33.2\%) followed by the Far East (91, $20 \%$ ), Central Asia (65, 14.3\%), Europe (51, 11.2\%) and Central and South America (47, 10.3\%). Most respondents have been practicing ophthalmology for more than 10 years. However, the distribution of the duration of clinical practice varied significantly $(p=0.020)$ between regions. The highest rate of physicians practicing over 15 years was reported in Central-Asia (96, 82.1\%) and lowest in Australia (8, 47.1\%). Overall 190 (41.8\%) respondents were affiliated to university hospitals with significant variation in prevalence between geographical regions $(p=0.004)$, ranging from $32.5 \%(49)$ in North-America to $56.3 \%(9)$ in Australia.

\section{Initiation of treatment}

Indications for initiation of treatment were described by 319 of respondents $(70 \%$ of respondents that treat myopia) and varied between regions (figure 2). The most common indication for treatment all over the world as well as in each geographical region was the rate of myopia progression (diopter/year (D/y)) $(239,75 \%$ of respondents). 


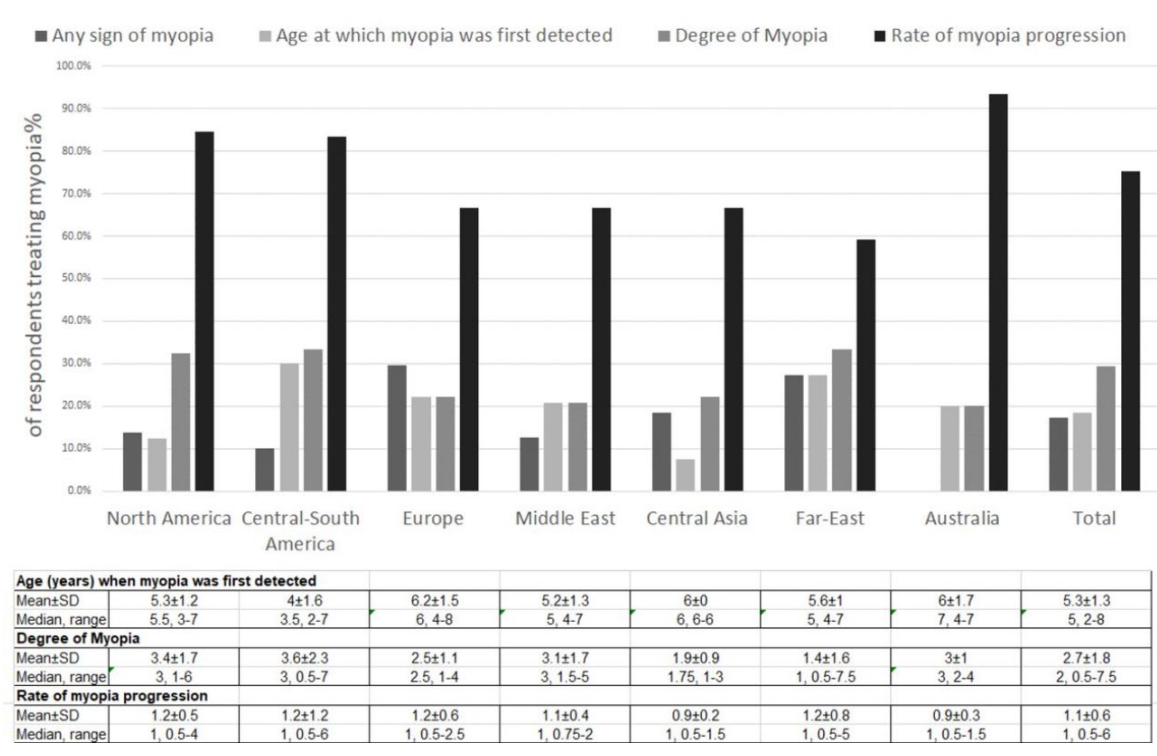

Figure 2

Variations between respondents from different geographical regions in implementation of criteria for initiation of treatment to reduce myopia progression.

On average, a progression rate of $1.1 \pm 0.6 \mathrm{D} / \mathrm{y}$ was chosen overall as the cut-off for initiation of treatment with minor deviations across regions (range $0.90-1.25, p=0.537$ ). Eighty-five respondents (27\%) started treatment when they identified a refractive error of $-2.7 \pm 1.8 \mathrm{D}$ or more. The degree of myopia to start treatment varied between regions. The cut-off was significantly $(p<0.05)$ lower among those from the Far East $(1.37 \pm 1.6)$ compared with North American (3.36 \pm 1.67$)$ and Central-South America $(-3.61 \pm 2.26)$ despite the similar prevalence in implementation of the criteria in those geographical regions (32\% and $33 \%$, respectively). The age cut-off did not differ significantly between regions (ANOVA, $\mathrm{p}=0.062$ ) with an overall mean of $5.33 \pm 1.35$ years. However, the prevalence of physicians who started treatment according to the age myopia was first detected differed between regions: $30 \%$ in Central-South America to $7.4 \%$ in Central Asia $(p=0.065)$.

\section{TREATMENT MODALITIES}

Most respondents treating myopia $(449,99 \%)$ selected the modality they considered to be most effective. Responses varied significantly between regions $(p<0.001)$. 
Pharmacological treatment was the most popular modality in most regions apart from Europe and Central Asia where optical treatment was preferred (figure 3). Respondents who initiated treatment when the rate of progression increased beyond their cut-off criteria selected pharmacological treatment at a significantly higher rate than those who did not choose that criteria $(81 \%$ vs $59 \%, p<0.001)$. In addition, the progression rate cut-off selected by physicians preferring the optical modality of treatment was significantly higher compared with the cut-off level chosen by those who opted for the pharmacological or behavioural treatment $(2.0 \pm 1.0 \mathrm{D} / \mathrm{y}$ vs $1.1 \pm 0.5$ and $1.1 \pm 0.4 \mathrm{D} / \mathrm{y}$, respectively, $p<0.001)$. Respondents who initiated treatment for any degree of myopia preferred pharmacological treatment significantly less than those who did not apply the level of myopia as a criterion $(57 \%$ vs $80 \%, p=0.002)$.

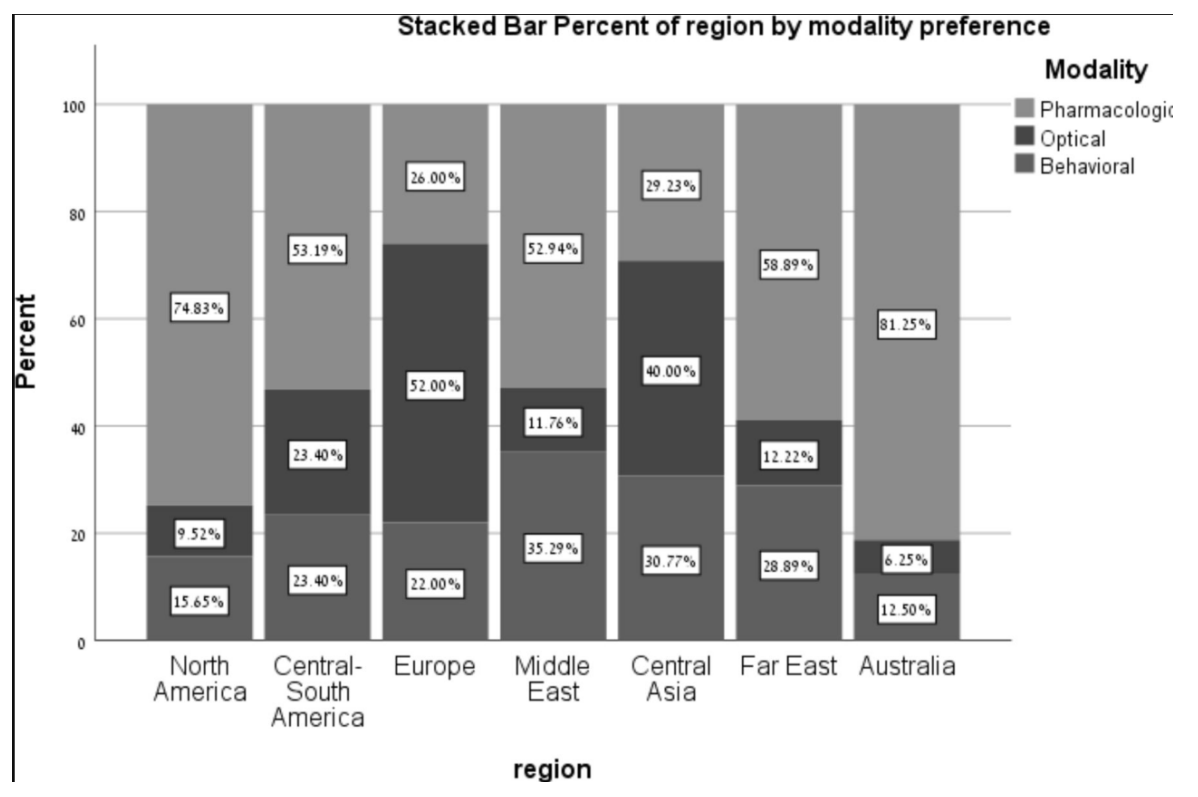

\section{Figure 3}

Variations between respondents from different geographical regions in the prevalence of modalities to reduce myopia progression.

Pharmacological treatment

Pharmacological treatment was used by $313(68.8 \%)$ respondents treating myopia progression. The prevalence of physicians using a pharmacological treatment varied significantly $(p<0.001)$ between regions (online supplementary table 3 ). Pharmacological 
treatment was significantly more popular among physicians treating myopia for 1-2 years or those with less than 15 years overall of clinical experience (online supplementary table 3).

Only pharmacological treatments with either atropine (all dosages) or pirenzepine were deemed effective (table 1).

The prevalence of physicians prescribing effective pharmacological treatments was $97 \%$ ( $n=303$ ) overall. It was $93 \%$ or higher in most regions, but lower in Europe (22/26, 85\%). An effective pharmacological treatment was prescribed by respondents who have chosen an optical treatment as the most effective modality at a significantly lower rate (20/26, $77 \%)$ compared with respondents who have chosen a behavioural modality (43/46, $93.5 \%)$ or a pharmacological modality $(236 / 237,99.6 \%)(p<0.001)$. Physicians who had initiated treatment according to a specific age at which myopia was first detected choose an effective treatment significantly less compared with those using other indications (50/55, $91 \%$ vs $248 / 253,98 \%, p=0.007)$. In contrast, physicians who initiated a treatment, when the rate of myopia progression exceeded a cut-off, used an effective treatment more than those using other indications $(228 / 233,98 \%$ vs $70 / 75,93 \%, p=0.055)$.

\section{OPTICAL TREATMENT}

The cohort included 405 (89\%) respondents using optical treatment to decrease myopia progression. The prevalence of physicians using optical treatment varied significantly among geographical regions $(p=0.010)$. It was most popular in Europe $(48,94.1 \%)$, Central-Asia (62, 95.4\%), Central-South America (45, 95.7\%) and the Far-East (84, $92.3 \%)$ and less popular in North-America $(126,83.4 \%)$, Australia $(12,75 \%)$ and the Middle-East $(28,82.4 \%)$. The prevalence was higher among respondents who were not affiliated to a university hospital $(243 / 265,91.7 \%$ vs $162 / 190,85.3 \%, p=0.030)$. The prevalence was also higher among those considering the optical modality to be the most effective $(92 / 93,98.9 \%)$ compared with respondents considering pharmacological $(214 / 251,85.35)$ or behavioural $(97 / 105,92.4 \%)$ modalities to be most effective, $\mathrm{p}=0.001)$. 
Overall, only 157 (38.8\%) of respondents using an optical treatment used an effective optical method. The prevalence of paediatric ophthalmologists using effective optical treatment varied significantly between regions (range $7 / 38,15.6 \%$ in Central-South America to $47 / 84,56 \%$ in the Far-East). Ophthalmologists with over 10 years of experience in myopia treatment used effective optical treatments at a significantly higher rate compared with less experienced ophthalmologists $(42 / 28,60 \%$ vs $112 / 332,33.7 \%$, $p<0.001)$. The use of an effective optical treatment was also significantly higher among those considering the optical modality to be most effective compared with physician who preferred one of the other two modalities $(49 / 92,53.3 \%$ vs $107 / 31134.4 \%, p=0.001)$. Additionally, there was a higher prevalence of physicians who offered an effective optical treatment among those that started treatment when myopia reached a certain threshold, in contrast to other criteria $(p=0.007)$.

\section{BEHAVIOURAL TREATMENT}

Among the three treatment modalities, behavioural treatment was used by the highest number of respondents $(414,92.2 \%)$. Again, the prevalence varied significantly between regions from $86 \%(130 / 151)$ in North-America to $98 \%(89 / 91)$ and $100 \%(65 / 65)$ in the Far-east and Central-Asia, respectively $(p=0.004)$. No other factors were found to significantly affect the prevalence of behavioural treatment implementation.

\section{COMBINATION OF MODALITIES}

Most respondents used a combination of either two $(174,38 \%)$ or three modalities of treatment $(255,56 \%)$. The histogram in figure 4 depicts the distribution of applied treatment modalities and combinations according to geographical regions. A combination of all three modalities was the most popular in most regions apart from Central-Asia where the prevalence of optical and behavioural combinations was higher. The optical and behavioural combination was the second most popular in most places excluding NorthAmerica and Australia. This combination was also used significantly more by physicians affiliated to an academic hospital (58/190, $30.5 \%$ vs $66 / 265,24.9 \%)$. In contrast, the triplemodality-combination was less popular among academically affiliated physicians (93/190, $48.9 \%$ vs $162 / 265,61.1 \%, p=0.018)$. The prevalence of triple-modality-combination was much higher among physicians who had deemed the pharmacological modality to be the 
most effective $(189 / 251,75.3 \%)$ compared with those who had opted for optical (23/93, $24.7 \%)$ or the behavioural $(41 / 105,39 \%)$ as most effective $(p<0.001)$. The distribution of the preference of treatment combination was not correlated with the amount of years in practice, experience in treating myopia progression or any specific indication for initiation of treatment.

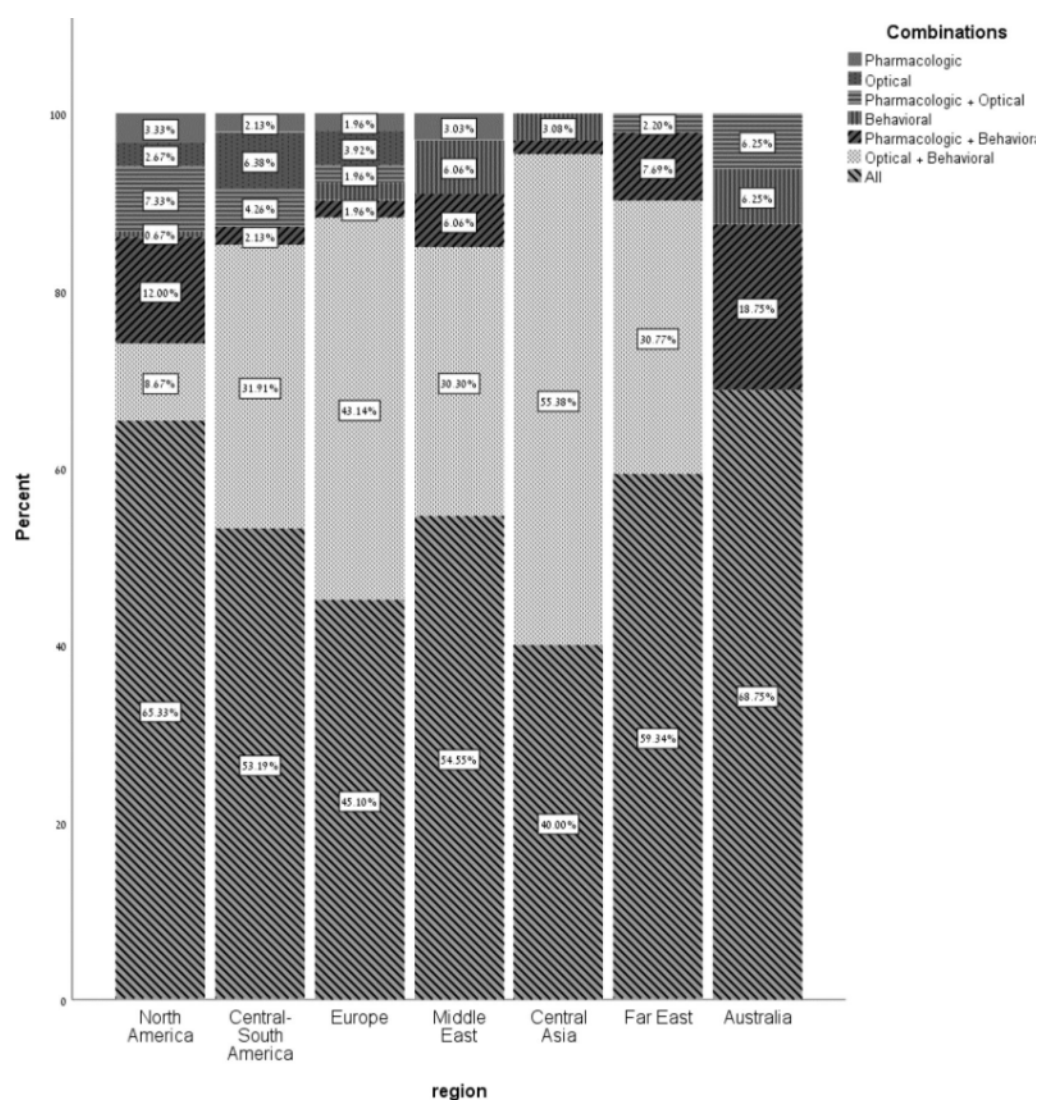

\section{Figure 4}

Variations between respondents from different geographical regions in prevalence of combinations of modalities to reduce myopia progression.

The prevalence of physicians using an effective pharmacological or optical treatment did not differ significantly whether they used a single or multiple treatment modalities.

\section{DISCUSSION}

Myopia is a growing concern among ophthalmologists worldwide. ${ }^{3}$ Although many types of medical interventions have been proposed to decrease myopia progression, only few 
have been proven to be effective. In this study, we attempted to describe compare between the real-life practice patterns of paediatric ophthalmologists around the world. Significant variability in myopia treatment choices was observed. It was affected by the geography, clinical experience and more specifically, experience in treating myopia progression.

According to our data, paediatric ophthalmologists who treat myopia progression were usually experienced physicians with over 15 years of clinical practice, although most have been treating myopia for less than 5 years. This discrepancy could be due to both the growing awareness of the myopia epidemic in recent years ${ }^{3}$ and the emergence of multiple studies regarding the various treatment options. These observations indicate that awareness of importance to treat myopia progression might be lacking both among young and seasoned paediatric ophthalmologist. Most treating physicians, who have responded to the questionnaire, were not affiliated to a university hospital, similar to the percentage among all the respondents. As previously reported by our group, $\underline{6}$ we observed a significant difference in treatment rates between geographical regions, yet this difference did not seem to be related to either affiliation or to number of years in practice.

Despite the lack of consensus in the literature when to initiate treatment, $\underline{5}$ the results from our survey show that the vast majority of physicians use a myopia progression rate of $\sim 1$ $\mathrm{D} / \mathrm{y}$ as the main guideline, with minor regional variations (figure 2). In contrast, there were greater geographical variations in the criteria based on the degree of myopia or the age myopia was first detected. This was evident both in prevalence of use and refractive or age cutoffs. Paediatric ophthalmologists from Central-Asia and the Far-East Asia chose a significantly lower myopic refractive error cut-off compared with all other regions. This might be due to the high prevalence of myopia in those regions. $\frac{31314}{14}$ The relatively high prevalence of initiation of treatment at the first sign of any degree of myopia in these regions support this practice pattern.

The use of pharmacological modalities varied significantly between regions. Overall, it was significantly more popular among newer physicians and among those who more recently started to treat myopia, probably due to a higher utility of educational material to improve knowledge among physician new to the field. These results may explain in part 
the relatively high prevalence of pharmacological treatment observed among physicians in North-America and Australia from which there was a high rate of respondents with a short experience in myopia treatment and few years of clinical practice. The vast majority of physicians in all the regions promoting a pharmacological treatment choose an effective pharmacological drug. Respondents from around the world, that chose pharmacological treatment as the most effective modality, used this modality effectively much more frequently. Pharmacological treatment was least popular among European physicians. Furthermore, less of them offered an effective pharmacological treatment compared with all the other regions ( $85 \%$ vs $93 \%)$. This might be due to the fact most studies were conducted in Asia in Chinese children, raising a concern these data might be less applicable to Caucasians. ${ }^{215-17}$ It should be noted that despite the growing evidence in the literature that supports the use of atropine to decrease myopic progression, $\frac{17-21}{17}$ pharmacological treatments were not used by almost a third of respondents treating myopia progression.

The popularity of employing optical treatments varied significantly between regions. The relatively lower rate among physicians from North-America and Australia corresponds to the higher rates of pharmacological treatments in these regions. More importantly, considerably less respondents prescribed effective optical treatments than effective pharmacological treatments, with significant variation between regions. It is apparent that higher rates of applied optic treatments did not necessarily mean a high rate of using an effective optical treatment (eg, a 95.7\% administration rate in Central-South America of which only $15.6 \%$ were effective). The large variation between regions and low rate of efficient treatment might stem from the lack of continued medical education (CME) of paediatric ophthalmologist regarding the best effective optical treatment alternatives. An additional factor is the availability and popularity of optical solutions that improve visual acuity without decreasing myopic progression, for example, monofocal eyeglasses instead of progressive addition lenses. Respondents who have selected optical modality as the most effect treatment option also significantly used optical treatments at a higher rate and choose a more effective treatment. The prevalence of offering an optical modality was significantly higher among physicians not affiliated to a university hospital. However, the rate of choosing effective optical treatments was similar between respondents who 
were affiliated to university hospitals and those who were not. Clinical experience was also associated with an increased rate of using effective optical treatments.

Behavioural treatment was the most popular modality used by over $90 \%$ of respondents. There is ample evidence regarding the association between behavioural risk factors (eg, increased near visual activity, sedentary posture or reduced time outdoors) and myopia progression. $\frac{1722-24}{24}$ The prevalence of the use of this modality was significantly higher in Far East Asia, where behavioural risk factors were shown to play a major role in myopia development. 17 Taking preventive measures might have made this modality popular as a first-line inexpensive and available treatment as opposed to both the pharmacological and optical modalities that offer interventional measures.

The majority of physicians across all regions used two or more modalities, most probably owing to the lack of consensus as to which modality is most effective and possible synergistic effects of these combinations. $\frac{25}{}$ We also observed a significantly higher prevalence of the combing pharmacological, optical and behavioural treatments by physicians who had deemed the pharmacological modality to be the most effective. This may suggest that these respondents were more familiar with current literature recommendations.

In conclusion, in this study, we found marked variation in the practice patterns to decrease myopia progression around the world. Although there are not any clear guidelines for initiation of treatment, our survey found a relative global consensus regarding initiating treatment when myopia progresses at a rate of $1 \mathrm{D} / \mathrm{y}$. Treatment selection of effective measures differed greatly between regions. The differences were most apparent in the use of optic treatments where a plethora of options most of which are ineffective lead to maltreatment to control myopia. In our survey, physicians who were familiar with pharmacological treatments also knew when to initiate treatment and used much more all three modalities. It is not surprising, however, that many respondents did not know how to effectively treat myopia progression, probably due to lack of CME. In order to alter the expanding myopia epidemic, we advocate that treatment recommendation criteria and guidelines be made by national as well as supranational medical organisations. Many 
more national public health programmes $\stackrel{26}{2}$ should advance and disseminate this information to practicing paediatric ophthalmologists by different means such as CME.

\section{REFERENCES}

1. Lin LL , Shih YF , Hsiao CK, et al . Prevalence of myopia in Taiwanese schoolchildren: 1983 to 2000. Ann Acad Med Singapore 2004;33:27-33.

2. Lam CS-Y, Lam C-H , Cheng SC-K, et al . Prevalence of myopia among Hong Kong Chinese schoolchildren: changes over two decades. Ophthalmic Physiol Opt 2012;32:17-24.doi:10.1111/j.1475-1313.2011.00886.x

3. Holden B , Mariotti S, Kocur I, et al . The impact of myopia and high myopia, 2015. Available: http://www.who.int/blindness/causes/MyopiaReportforWeb.pdf

4. Huang $J$, Wen $D$, Wang $Q$, et al . Efficacy comparison of 16 interventions for myopia control in children: a network meta-analysis. Ophthalmology 2016;123:697708.doi:10.1016/j.ophtha.2015.11.010

5. Pineles SL , Kraker RT, VanderVeen DK, et al . Atropine for the prevention of myopia progression in children: a report by the American Academy of ophthalmology. Ophthalmology 2017;124:1857-66.doi:10.1016/j.ophtha.2017.05.032

6. Zloto $\mathrm{O}$, Wygnanski-Jaffe $\mathrm{T}$, Farzavandi SK, et al . Current trends among pediatric ophthalmologists to decrease myopia progression-an international perspective. Graefes Arch Clin Exp Ophthalmol 2018;256:2457-66.doi:10.1007/s00417-018-4078-6

7. He X , Sankaridurg $P$, Xiong $S$, et al . Shanghai time outside to reduce myopia trial: design and baseline data. Clin Exp Ophthalmol 2019;47:171-8.doi:10.1111/ceo.13391

8. Pärssinen $O$, Kauppinen $M$, Viljanen $A$. The progression of myopia from its onset at age 8-12 to adulthood and the influence of heredity and external factors on myopic progression. A 23-year follow-up study. Acta Ophthalmol 2014;92:7309.doi:10.1111/aos.12387

9. Li S-M , Li H , Li S-Y, et al . Time outdoors and myopia progression over 2 years in Chinese children: the Anyang childhood eye study. Invest Ophthalmol Vis Sci 2015;56:4734-40.doi:10.1167/iovs.14-15474 
10. Jin J-X, Hua W-J, Jiang $X$, et al . Effect of outdoor activity on myopia onset and progression in school-aged children in northeast China: the sujiatun eye care study. BMC Ophthalmol 2015;15:1-11.doi:10.1186/s12886-015-0052-9

11. Wu P-C , Chen C-T , Lin K-K , et al . Myopia prevention and outdoor light intensity in a school-based cluster randomized trial. Ophthalmology 2018;125:1239_ 50.doi:10.1016/j.ophtha.2017.12.011

12. Guo Y, Liu LJ , Tang P , et al . Outdoor activity and myopia progression in 4-year followup of Chinese primary school children: the Beijing children eye study. PLoS One 2017;12:e0175921-14.doi:10.1371/journal.pone.0175921

13. Wong TY, Ferreira A, Hughes $R$, et al . Epidemiology and disease burden of pathologic myopia and myopic choroidal neovascularization: an evidence-based systematic review. Am J Ophthalmol 2014;157:9-25.doi:10.1016/j.ajo.2013.08.010

14. Saxena R, Vashist $P$, Tandon $R$, et al . Incidence and progression of myopia and associated factors in urban school children in Delhi: the North India myopia study (nim study). PLoS One 2017;12:e0117349.doi:10.1371/journal.pone.0189774

15. Chia A , Chua W-H , Cheung Y-B , et al . Atropine for the treatment of childhood myopia: safety and efficacy of $0.5 \%, 0.1 \%$, and $0.01 \%$ doses (Atropine for the Treatment of Myopia 2). Ophthalmology 2012;119:347-54.doi:10.1016/j.ophtha.2011.07.031

16. Chua W-H , Balakrishnan V, Chan Y-H , et al . Atropine for the treatment of childhood myopia. Ophthalmology 2006;113:2285-91.doi:10.1016/j.ophtha.2006.05.062

17. Morgan IG , French AN , Ashby RS, et al . The epidemics of myopia: aetiology and prevention. Prog Retin Eye Res 2018;62:134-49.doi:10.1016/j.preteyeres.2017.09.004

18. Polling JR, Kok RGW, Tideman JWL, et al. Effectiveness study of atropine for progressive myopia in Europeans. Eye 2016;30:998-1004.doi:10.1038/eye.2016.78

19. Pineles SL , Kraker RT, VanderVeen DK, et al . Atropine for the prevention of myopia progression in children. Ophthalmology 2017;124:185766.doi:10.1016/j.ophtha.2017.05.032

20. Tan D , Tay SA, Loh K-L, et al . Topical atropine in the control of myopia. Asia Pac J Ophthalmol 2016;5:424-8.doi:10.1097/APO.0000000000000232 
21. Leo SW , Scientific Bureau of World Society of Paediatric Ophthalmology and Strabismus (WSPOS) . Current approaches to myopia control. Curr Opin Ophthalmol 2017;28:267-75.doi:10.1097/ICU.0000000000000367

22. French AN , Morgan IG, Mitchell P, et al . Risk factors for incident myopia in Australian schoolchildren: the Sydney adolescent vascular and eye study. Ophthalmology 2013;120:2100-8.doi:10.1016/j.ophtha.2013.02.035

23. Rose KA, Morgan IG, Ip J, et al. Outdoor activity reduces the prevalence of myopia in children. Ophthalmology 2008;115:1279-85.doi:10.1016/j.ophtha.2007.12.019

24. Ku P-W , Steptoe A, Lai Y-J , et al . The associations between near visual activity and incident myopia in children: a nationwide 4-year follow-up study. Ophthalmology 2019;126:214-20.doi:10.1016/j.ophtha.2018.05.010

25. Wan L, Wei C-C, Chen C, et al . The synergistic effects of Orthokeratology and atropine in slowing the progression of myopia. $\mathrm{J}$ Clin Med 2018;7.doi:10.3390/jcm7090259

26. Seet B , Wong TY, Tan DT , et al . Myopia in Singapore: taking a public health approach. Br J Ophthalmol 2001;85:521-6.doi:10.1136/bjo.85.5.521 